\title{
Editorial: Hybrid Intelligent Algorithms Based Learning, Optimization, and Application to Autonomic Control Systems
}

\author{
Yanzheng Zhu ${ }^{1 *}$, Hak-Keung Lam ${ }^{2}$, Ting Yang ${ }^{3}$, Zhixiong Zhong ${ }^{4}$ and Sabri Arik ${ }^{5}$ \\ ${ }^{1}$ College of Mechanical Engineering and Automation, Huaqiao University, Xiamen, China, ${ }^{2}$ Department of Informatics, King's \\ College London, London, United Kingdom, ${ }^{3}$ School of Automation, Northwestern Polytechnical University, Xi'an, China, \\ ${ }^{4}$ College of Computer and Control Engineering, Minjiang University, Fuzhou, China, ${ }^{5}$ Department of Computer Engineering, \\ Faculty of Engineering, Istanbul University-Cerrahpasa, Istanbul, Turkey
}

Keywords: artificial intelligence, autonomous systems, intelligent control, machine learning, optimization

\section{Editorial on the Research Topic}

Hybrid Intelligent Algorithms Based Learning, Optimization, and Application to Autonomic Control Systems

With the rapid rise of artificial intelligence, a large amount of intelligent techniques, including neural networks (Zhang et al., 2015), fuzzy logic (Zhong et al., 2017), and genetic algorithms (Rovithakis et al., 2004) have been broadly applied to various fields, such as chemical process, robotics, mechanical engineering, etc. In biological systems, the neural networks usually contain a

OPEN ACCESS

Edited and reviewed by: Vaughan G. Macefield,

Baker Heart and Diabetes Institute, Australia

*Correspondence: Yanzheng Zhu yanzhengzhu@hqu.edu.cn

Specialty section:

This article was submitted to Autonomic Neuroscience, a section of the journal Frontiers in Neuroscience

Received: 06 September 2019 Accepted: 27 September 2019 Published: 11 October 2019

Citation:

Zhu Y, Lam H-K, Yang T, Zhong Z and Arik S (2019) Editorial: Hybrid Intelligent Algorithms Based Learning,

Optimization, and Application to Autonomic Control Systems.

Front. Neurosci. 13:1090. doi: 10.3389/fnins.2019.01090 finite set of modes that switch in accordance with internal evolution and external stimulation, and such switching can often be represented as stochastic (Zhang et al., 2016) or even non-deterministic form (Yang and Zheng, 2018). Recent novel developments in the system and control community on control and filtering of intelligent systems with some hybrid switching characteristics. However, the practical applications in the areas of tele-medicine, disease treatment, and healthcare are lacking due largely to the limitations of existing hybrid intelligent systems. It is also difficult and challenging to implant these hybrid intelligent algorithms into the process of existing manufacturing facilities and equipment research and development.

Recently, autonomous control (Antsaklis et al., 1991; Pachter and Chandler, 1998) - based on the mode of operation of the autonomic nervous system-has emerged with the advent of artificial intelligence, and an ever-increasing demand has been placed by users in different fields (Isakhani et al., 2018; Shen et al., 2018). It is expected that the advanced intelligent algorithms can be fitted into the learning, optimization and control design to improve autonomous ability. Also, exploration of the novel communication mechanisms between autonomous systems and other regulatory systems is very welcome, building on the existing approaches on networked control systems with communication constraints (Heemels et al., 2010). The current Research Topic provides a useful overview of hybrid intelligent algorithm-based learning, optimization and the applications of these new autonomous control strategies.

Wang et al. investigate the stability and stabilization control problem for non-linear T-S fuzzy sampled-data systems under time-varying sampling intervals, with and without quantized states. A new Lyapunov-Krasoskii functional (LKF) named discontinuous LKF is constructed, such that the LKF is not necessary to be positive all the time, but only positive at the sampling time. By using the proposed discontinuous LKF approach and free-matrix-based integral inequality boundary processing technique, stability conditions that are less conservative are obtained for T-S fuzzy 
systems with and without sampled-data quantized states, and the required sampled-data controllers are designed simultaneously. The simulation results show that the maximum sampling interval of T-S fuzzy sampling-data systems with and without quantized states are both larger than the existing results.

Zhang et al. establish the periodic event-triggered control (PETC) scheme of robust $H_{\infty}$ filtering for a class of uncertain discrete-time Takagi-Sugeno (T-S) fuzzy systems, where the sample time is assumed to be a constant. Two frameworks are introduced based on perturbed linear and piecewise linear systems to analyze the filtering problems of the PETC strategy. Sufficient conditions for the existence of a robust $H_{\infty}$ filter are derived in the form of matrix inequalities. The effectiveness of the proposed approach is testified by using a simulation example.

Guan et al. propose a robust adaptive recurrent cerebellar model articulation controller (RCMAC) for non-linear MIMO systems, where the GPSO-based RCMAC with the adaptive law is used for updating parameters, and the learning rates can be optimized to best value based on the GPSO algorithm. It has been shown that the proposed robust controller can be designed to compensate for the difference between adaptive RCMAC and ideal controller.

Zhao et al. present the wavelet fuzzy brain emotional learning controller (WFBELC) model for the uncertainty of the MIMO non-linear systems based on the wavelet theory, type-1 fuzzy inference and the BEL algorithm. The WFBELC is used as the main tracking controller for a MIMO uncertain non-linear system and the robust compensation controller is used as a compensator. It has been shown that this proposed WFBELC model can effectively obtain satisfactory control capability with better transient responses and smaller error values, compared to the fuzzy cerebellar model articulation controller (FCMAC) design scheme and the brain emotional learning controller (BELC) design scheme.

\section{REFERENCES}

Antsaklis, P. J., Passino, K. M., and Wang, S. J. (1991). An introduction to autonomous control systems. IEEE Control Syst. Magaz. 11, 5-13. doi: $10.1109 / 37.88585$

Heemels, W. P. M. H., Teel, A. R., Van de Wouw, N., and Nesic, D. (2010). Networked control systems with communication constraints: Tradeoffs between transmission intervals, delays and performance. IEEE Trans. Automatic Control 55, 1781-1796. doi: 10.1109/TAC.2010.2042352

Isakhani, H., Aouf, N., Stamatis, O. K., and Whidborne, J. F. (2018). A furcated visual collision avoidance system for an autonomous micro robot. IEEE Trans. Cogn. Dev. Syst. doi: 10.1109/TCDS.2018.2858742. [Epub ahead of print].

Pachter, M., and Chandler, P. R. (1998). Challenges of autonomous control. IEEE Control Syst. Magaz. 18, 92-97. doi: 10.1109/37.710883

Rovithakis, G., A., Maniadakis, M., and Zervakis, M. (2004). A hybrid neural network/genetic algorithm approach to optimizing feature extraction for signal classification. IEEE Trans. Syst. Man Cybern. Part B 34, 695-703. doi: 10.1109/TSMCB.2003.811293

Shen, C., Shi, Y., and Buckham, B. (2018). Trajectory tracking control of an autonomous underwater vehicle using Lyapunov-based model predictive control. IEEE Trans. Indus. Electron. 65, 5796-5805. doi: 10.1109/TIE.2017.2779442

Yang, R., and Zheng, W. X. (2018). $H_{\infty}$ filtering for discrete-time 2-D switched systems: an extended average dwell time approach. Automatica 98, 302-313. doi: 10.1016/j.automatica.2018.09.013
Bi et al. introduce a novel model called the GeneticEvolutionary Random Support Vector Machine Cluster (GERSVMC) to classify individuals with Asperger syndrome and neurotypical individuals, and search for lesions within the brain. The model innovatively integrates the methods of cluster and genetic evolution to improve the performance of the model. It has been shown that the classification accuracy of the model reaches $97.5 \%$ and identifies brain regions showing significant differences. The proposed method also provides a new perspective for the diagnosis and treatment of Asperger syndrome.

\section{AUTHOR CONTRIBUTIONS}

All authors listed have made a substantial, direct and intellectual contribution to the work, and approved it for publication.

\section{FUNDING}

This special issue has been supported in part by National Natural Science Foundation of China $(61603221,61973131)$, and in part by the Scientific Research Funds of Huaqiao University (60550Y19010).

\section{ACKNOWLEDGMENTS}

All of the topic editors would like to thank all authors for providing high-quality manuscripts on this Research Topic in a very timely fashion. We are also indebted to the referees for their time and valuable comments, criticism, and suggestions. Finally, we very much appreciate their support, advice, and assistance of Journal Development Specialists Alessia Luoni and Eleni Batzianouli, and our Frontiers in Neuroscience team to successfully organize this Research Topic.

Zhang, L., Yang, T., and Patrizio, C. (2016). Stability and stabilization of semi-Markov jump linear systems with exponentially modulated periodic distributions of sojourn time. IEEE Trans. Automatic Control 62, 2870-2885. doi: 10.1109/TAC.2016.26 18844

Zhang, L., Zhu, Y., and Zheng, W. X. (2015). Synchronization and state estimation of a class of hierarchical hybrid neural networks with timevarying delays. IEEE Trans. Neural Netw. Learn. Syst. 27, 459-470. doi: 10.1109/TNNLS.2015.2412676

Zhong, Z., Zhu, Y., and Lam, H. K. (2017). Asynchronous piecewise output-feedback control for large-scale fuzzy systems via distributed event-triggering schemes. IEEE Trans. Fuzzy Syst. 26, 1688-1703. doi: 10.1109/TFUZZ.2017.2744599

Conflict of Interest: The authors declare that the research was conducted in the absence of any commercial or financial relationships that could be construed as a potential conflict of interest.

Copyright (c) 2019 Zhu, Lam, Yang, Zhong and Arik. This is an open-access article distributed under the terms of the Creative Commons Attribution License (CC BY). The use, distribution or reproduction in other forums is permitted, provided the original author(s) and the copyright owner(s) are credited and that the original publication in this journal is cited, in accordance with accepted academic practice. No use, distribution or reproduction is permitted which does not comply with these terms. 